Hyan de Alvarenga Moreira ${ }^{a}$

(D) Orcid: https://orcid.org/0000-0002-6574-7143

Karen Nattana de Souza ${ }^{\text {a }}$

(iD) Orcid: https://orcid.org//0000-0002-1691-9834

Mirian Ueda Yamaguchi ${ }^{b}$

iD Orcid: https://orcid.org/0000-0001-5065-481X

a Centro Universitário de Maringá, Curso de Graduação em Medicina. Maringá, Paraná, Brasil.

b Centro Universitário de Maringá, Instituto Cesumar de Ciência, Tecnologia e Inovação. Mestrado em Promoção da Saúde. Maringá, Paraná, Brasil.

Contato:

Hyan de Alvarenga Moreira

E-mail:

ham.0060@gmail.com

Pesquisa desenvolvida a partir do

Trabalho de Conclusão do Curso de Graduação em Medicina, de autoria de Hyan de Alvarenga Moreira, Karen Nattana de Souza e Mirian Ueda Yamaguchi, sob o título "Síndrome de Burnout em médicos: uma revisão sistemática", apresentado ao Centro Universitário de Maringá (UniCesumar), Maringá/PR, em 2015.

Os autores declaram que o trabalho não foi subvencionado e que não há conflitos de interesses.

Os autores informam que o trabalho não foi apresentado em eventos científicos.

\section{Síndrome de Burnout em médicos: uma revisão sistemática}

\author{
Burnout syndrome in physicians: \\ a systematic review
}

\begin{abstract}
Resumo
Introdução: médicos são profissionais altamente vulneráveis à Síndrome de Burnout (SB), pois lidam com fatores de stress ao longo de sua vida profissional. Objetivo: identificar a frequência da SB em diferentes especialidades médicas e seus possíveis fatores associados. Métodos: revisão sistemática de acordo com a declaração PRISMA, por meio das bases de dados LILACS, MEDLINE, PubMed e SciELO, incluindo trabalhos que usam o Maslach Burnout Inventory para identificar a síndrome. Resultados: 22 especialidades médicas foram relacionadas à SB. As cinco especialidades identificadas com maior prevalência de casos foram, em ordem decrescente: medicina de Unidade de Terapia Intensiva, medicina de família, medicina de emergência, medicina interna e ortopedia. Discussão: a etiologia da SB é multifatorial. Há fatores que contribuem para o desencadeamento da síndrome e outros que atuam de modo a prevenir o seu desenvolvimento. Identificaram-se fatores comuns a atuação profissional do médico e outros associados com as especialidades médicas. Os fatores que se destacam como associados à SB são os relacionados à organização e ambiente do trabalho e à maneira como os profissionais enfrentam o stress.
\end{abstract}

Palavras-chave: esgotamento profissional; médicos; revisão; qualidade da assistência à saúde.

\begin{abstract}
Introduction: physicians are highly vulnerable to Burnout Syndrome (BS) because they deal with stressors throughout their professional lives. Objective: to identify the frequency of BS in different medical specialties and its possible associated factors. Methods: systematic review according to the PRISMA statement, using LILACS, MEDLINE, PubMed and SciELO databases, and selecting studies that use the Maslach Burnout Inventory to identify and quantify the syndrome. Results: 22 medical specialties were identified as related to $B S$. The five specialties with the highest prevalence of BS cases were, in decreasing order: intensive care unit medicine, family medicine, emergency medicine, internal medicine and orthopedics. Discussion: the syndrome etiology is multifactorial. There are factors associated with BS that contribute to the onset of the syndrome and others that may prevent its outcome. Factors that are common to medical practice and others associated with medical specialties were identified. The main factors associated with BS are those related to work organization and environment and the way physicians cope with stress.
\end{abstract}

Keywords: burnout; physicians; review; health care quality. 


\section{Introdução}

Derivado do inglês "to burn out" ("queimar-se, consumir-se" em português), o termo Síndrome de Burnout (SB) foi usado por primeira vez, em 1974, pelo psicanalista Herbert Freudenberger ${ }^{1}$ ao observar que seu trabalho não lhe trazia o mesmo prazer de outrora, relacionando a sensação de esgotamento à falta de estímulo originado da escassez de energia emocional. Além desses sintomas, Freudenberger incluiu fadiga, depressão, irritação e inflexibilidade como pertencentes ao quadro sintomatológico da SB. Em 1981, Christina Maslach e Susan Jackson ${ }^{2}$ colocaram a SB na perspectiva de um stress intenso e contínuo provocado pelo trabalho. Em 1999, Christina Maslach e Michael Leiter ${ }^{3}$ deram à SB sua definição e caracterização final: uma síndrome composta pelos tripés exaustão emocional, despersonalização e falta de realização profissional ${ }^{4}$.

No que diz respeito aos subitens do tripé que compõem a SB, o emocional esgotado se refere a sentimentos de cansaço, em que não há mais energia emocional suficiente. A despersonalização, nesta síndrome, engloba um distanciamento na relação com os pacientes e colegas de trabalho, além de uma baixa empatia para com o outro; e a desvalia profissional é caracterizada por uma sensação de incapacidade produtiva, de autoestima diminuída em relação ao trabalho ${ }^{5}$.

Para avaliar esses subitens da SB, o instrumento mais utilizado é o Maslach Burnout Inventory (MBI) criado por Maslach e Jackson, sendo considerado o mais fidedigno, pois avalia diferentes subitens em comparação com os outros questionários que analisam apenas $u^{6}$. Trata-se de um questionário com 22 questões a serem respondidas sobre sentimentos em relação ao trabalho e à frequência dos sintomas, pontuando os resultados. Para os subitens que compõem a síndrome (exaustão emocional - EE, despersonalização - DP e realização profissional - RP), há 9, 5 e 8 questões, respectivamente. Cada subitem é avaliado em baixo, médio e alto. Em relação à EE, uma pontuação baixa é aquela $\leq 14$; um score médio é aquele compreendido entre 15 e 24 ; e alto com resulta$\operatorname{dos} \geq 25$. Para o subitem DP, a pontuação $\leq 3$ significa baixo índice, entre 4 a 9 é médio e $\geq 10$, alto. Por fim, para RP, pontuações $\geq 40$ indicam baixo índice (pois a escala é inversa às demais), entre 33 a 39 pontos é um índice médio e $\leq 32$ é alto ${ }^{6,7}$. Alta pontuação nos subitens desgaste emocional e despersonalização, e baixa pontuação no subitem realização profissional, é classificado como alto índice da $\mathrm{SB}^{8,9}$. O desenvolvimento da síndrome ocorre por meio de um processo dinâmico que envolve os três subitens EE, DP e RP, em que inicialmente ocorre uma elevação dos três e, em sequência, há redução da EE e progressão para os níveis graves de DP e RP. Porém, um item alterado isoladamente não é suficiente para definir a $\mathrm{SB}^{3}$. A SB está incluída no capítulo XXI do CID-10, ao qual se refere a problemas relacionados com a organização de seu modo de vida, identificada com o código Z73.0 ${ }^{10}$. No Brasil, em 1999 o Ministério da Saúde a incluiu na lista de Doenças relacionadas ao trabalho por meio da Portaria $\mathrm{n}^{\circ}$ 1.339. Em 2007, foi inserida na Lista B da Previdência Social, sob título Transtornos mentais e do comportamento relacionados com o trabalho, por meio do Decreto $\mathrm{n}^{\mathrm{o}} 6.042^{11}$. Em relação à discussão sobre SB no Brasil, iniciou-se com a publicação do médico cardiologista Hudson Hubner França em 1987 na Revista Brasileira de Medicina, segundo BenevidesPereira $^{12}$. Em 2016, de acordo com pesquisa feita no Diretório dos Grupos de Pesquisa no Brasil, disponível na plataforma Lattes do CNPq (Conselho Nacional de Desenvolvimento Científico e Tecnológico), estavam cadastrados 14 grupos de pesquisa relacionados à SB.

Nesse contexto, a SB é um problema de saúde pública que pode acarretar ausência no trabalho e licença por doença, gerando despesa para a organização empregadora, além de afetar a qualidade do serviço oferecido, a produtividade e o lucro ${ }^{13}$.

Profissões que demandam alto nível de stress no dia a dia são mais susceptíveis à SB, particularmente nos profissionais da área da saúde ${ }^{14-16}$. Especificamente na área da Medicina a presença desta síndrome é crítica: a nível mundial está presente em 1 a cada 2 médicos; um terço destes é afetado de maneira considerável; e um décimo, de forma grave com aspectos irremediáveis ${ }^{17,18}$. De acordo com o Conselho Federal de Medicina ${ }^{19}$, no Brasil 23,1\% dos médicos apresentam a SB em alto grau em uma amostra de 7,7 mil profissionais de todos os estados.

Lima et al. citam que a SB pode ser desencadeada pela presença de stress emocional contínuo, destacando-se: o constante cuidar de pessoas e a responsabilidade do profissional médico; o cenário atual, em que ocorre uma pressão cada vez maior para a incorporação de condutas e procedimentos que visam ao melhor desempenho dos médicos na produção e a desvalorização profissional que essa área vem sofrendo perante a sociedade, fato observado na hostilidade ocorrida no cotidiano do trabalho dos médicos, em que muitas vezes os pacientes procuram a Justiça como resultado de descontentamento por determinado serviço ${ }^{20}$.

Outros fatores emocionais são entendidos ${ }^{5}$ como desencadeantes de stress no médico, como a dor, o medo e a angústia, que podem envolver o profissional no trabalho com um paciente grave ou mesmo com seus familiares. Ou ainda, a sensação de frustração ou impotência quando ocorrem evoluções negativas de quadro clínico de um paciente com um grave 
acometimento. Ainda assim, há outros agentes geradores de stress relacionados ao trabalho: a divisão da carga trabalhista por turnos, o salário insatisfatório e o ambiente ocupacional de emoções negativas. Acrescenta-se também a alta carga horária dos profissionais, o número reduzido de trabalhadores durante o expediente e o contato do médico com riscos biológicos, químicos e físicos no ambiente de trabalho ${ }^{21}$.

Ainda há incertezas sobre a prevalência da SB em médicos de vários países, além de que muitos profissionais da saúde desconhecem a existência da doença ou simplesmente a ignoram. Deste modo, é necessário que novos estudos sejam feitos para mensurar a taxa da SB entre os profissionais médicos. A divulgação desta síndrome entre os profissionais da saúde é essencial para que seja possível o reconhecimento dos sintomas, a consciência de que a SB difere de outras doenças psiquiátricas como depressão ${ }^{22}$ e a busca por tratamento ${ }^{13,23}$.

O objetivo deste trabalho foi identificar a frequência da SB de alto grau em diferentes especialidades médicas no mundo e possíveis fatores associados, a partir da análise dos artigos selecionados e incluídos na pesquisa.

\section{Métodos}

Este trabalho se fundamentou em revisão sistemática da literatura científica nacional e internacional (Figura 1) sobre o tema Síndrome de Burnout em médicos, cujo objeto de análise foi a produção científica veiculada em periódicos indexados nos bancos de dados da Literatura Latino-Americana e do Caribe em Ciências da Saúde (LILACS), National Library of Medicine (MEDLINE) e Scientific Electronic Library Online (SciELO), disponíveis na Biblioteca Virtual em Saúde - BVS (http://www.bireme.br), e também da United States National Library of Medicine - PubMed (http://www.ncbi.nlm.nih.gov/pubmed). A pesquisa foi realizada conforme recomendações metodológicas da declaração PRISMA (Preferred Reporting Items for Systematic Reviews and Meta-Analyses) para trabalhos de revisão sistemática ${ }^{24,25}$.

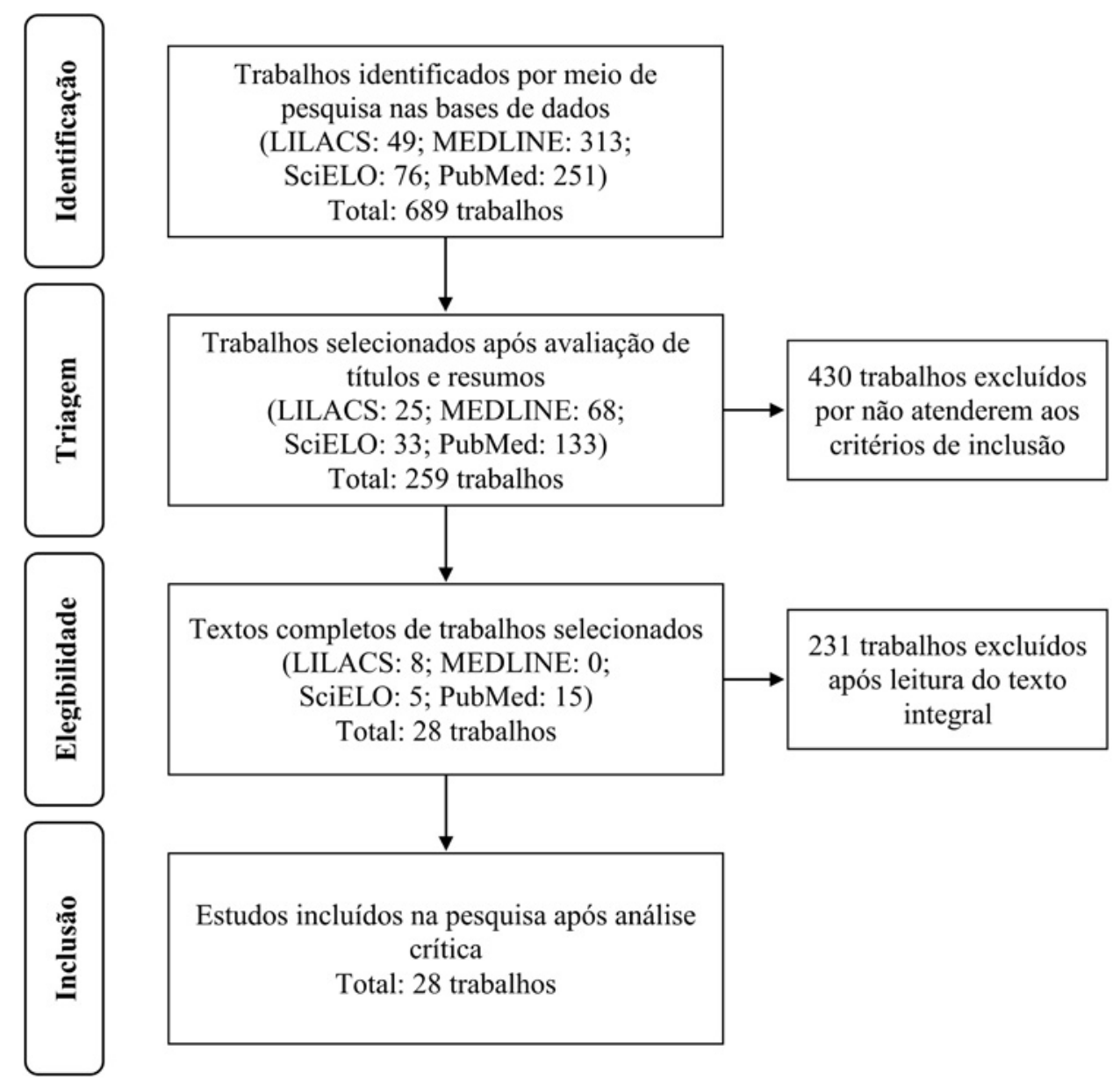

Figura 1 Representação esquemática dos métodos de identificação, triagem, elegibilidade e inclusão de trabalhos na revisão, adaptada de acordo com o PRISMA Flow Diagram 
A busca dos documentos ocorreu nos meses entre julho e agosto de 2015, e para isso foram usados os seguintes descritores: Maslach Burnout Inventory, médicos; e seus correspondentes em inglês (Maslach Burnout Inventory, physicians) e em espanhol (Maslach Burnout Inventory, médicos). Nas bases de dados LILACS, MEDLINE e SciELO foram aplicados os filtros: artigo e texto disponível. Já na base de dados PubMed foram aplicados os filtros: review, scientific integrity review, systematic reviews e full text available.

Por meio da busca, inicialmente foram rastreados 689 documentos, sendo que 251 foram encontrados na base de dados PubMed; e os demais, nas bases MEDLINE (313), LILACS (49) e SciELO (76). Em seguida, foram selecionados os trabalhos científicos incluídos no estudo por meio de avaliação dos títulos e resumos, de forma independente por dois pesquisadores, obedecendo aos seguintes critérios de inclusão: publicações compreendidas nos últimos 19 anos, de janeiro de 1996 a agosto de 2015; que utilizaram o instrumento Maslach Burnout Inventory para quantificar a SB; que incluíram profissionais médicos e que foram publicados nos idiomas português, inglês e espanhol.

Um desafio encontrado neste trabalho foi identificar, entre as pesquisas científicas publicadas, a inclusão dos médicos nos grupos de profissionais de saúde, visto que parte dos artigos informaram no título e resumo que a pesquisa foi realizada em funcionários da saúde ou em áreas hospitalares específicas, somente. Dessa forma, o critério de seleção permitiu incluir apenas os trabalhos que especificaram a inclusão de médicos no objeto de pesquisa e permitiram analisar os dados distintamente dos demais profissionais da saúde.
Os seguintes critérios de exclusão foram utilizados: trabalhos que utilizam escalas psicométricas para avaliar outros distúrbios psiquiátricos que interferem na identificação da SB; trabalhos que não forneceram resultados absolutos ou percentuais da prevalência da síndrome; assim como aqueles que incluíram vários profissionais não-médicos ou médicos de diferentes especialidades no estudo e não os discriminam nos resultados; artigos que não expuseram o resultado do MBI em suas três dimensões (EE, DP e RP) e artigos que analisam somente os internos de medicina, somente residentes ou médicos praticantes de uma área médica sem ser especialistas, exceto os clínicos gerais e médicos de família.

Após avaliação dos títulos e resumos, foram selecionados 259 trabalhos, sendo que destes, após leitura integral e aplicados os critérios de exclusão, 28 foram incluídos. Oito artigos, indisponíveis na versão on-line, foram também excluídos da revisão.

Por fim, depois da análise crítica dos artigos selecionados, realizada por dois pesquisadores de forma independente, os trabalhos foram classificados por autor, ano da publicação, local da pesquisa, objetivos, tipo de metodologia empregada e resultados apresentados.

\section{Resultados}

Em relação aos 28 artigos selecionados, a Figura 2 mostra o número de artigos incluídos, identificados nas bases de dados PubMed, LILACS e SciELO segundo o ano de publicação, no período compreendido entre 1996 e agosto de 2015.

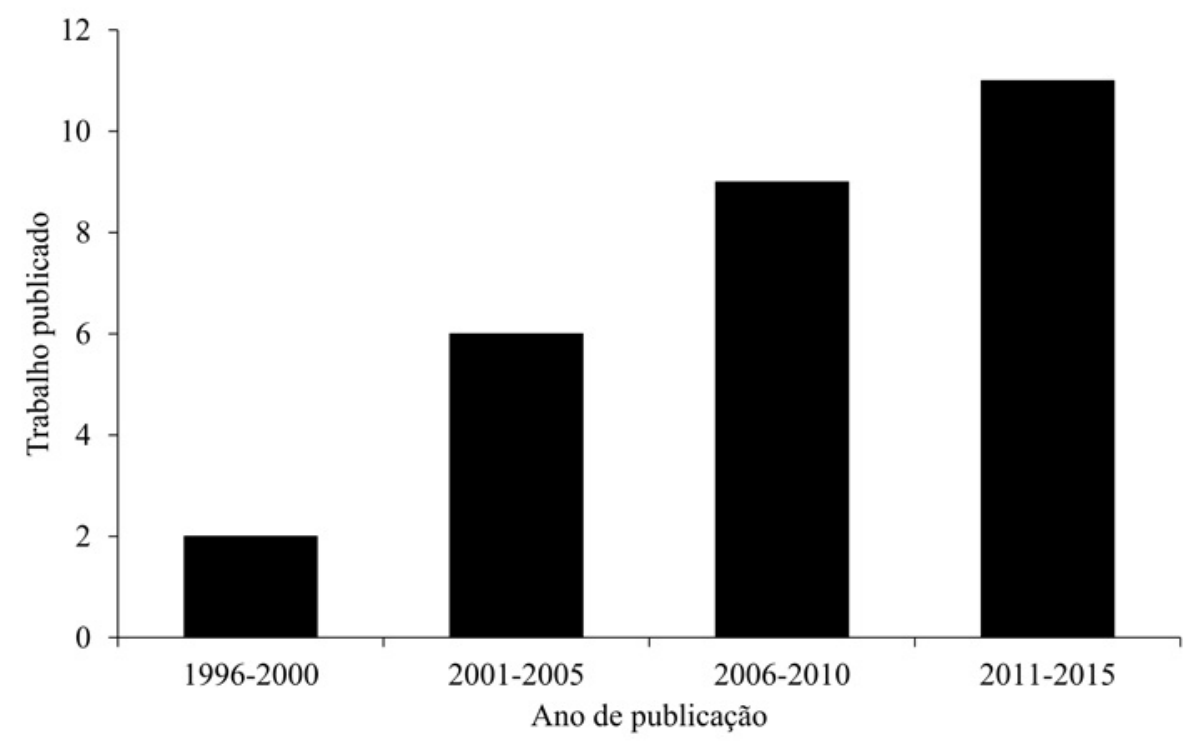

Figura 2 Número de trabalhos incluídos neste estudo publicados entre janeiro de 1996 e agosto de 2015 
Foram descritos os dados dos 28 artigos (Tabela 1), em que são expostos: autor principal, ano da publicação, países da pesquisa e as especialidades médicas estudadas. Do total de artigos incluídos na revisão, 8 continham dados do México, 7 da Espanha, 6 do Brasil, 3 da Argentina, 3 dos Estados Unidos (EUA), 2 da Holanda, 2 do
Reino Unido, 2 da Suécia, e cada um dos países a seguir esteve citado em 1 artigo: Austrália, Bélgica, Bulgária, Canadá, Colômbia, Croácia, Dinamarca, El Salvador, Equador, Finlândia, França, Grécia, Guatemala, Hungria, Islândia, Israel, Itália, Malta, Noruega, Nova Zelândia, Peru, Polônia, Portugal, Turquia e Uruguai.

Tabela 1 Artigos incluídos neste estudo, classificados segundo autor principal, ano da publicação, país da pesquisa e especialidades médicas do estudo

\begin{tabular}{|c|c|c|c|}
\hline Autor & Ano & País & Especialidades \\
\hline Goldberg et al. ${ }^{26}$ & 1996 & EUA & Emergências \\
\hline Arriortua et al. ${ }^{27}$ & 2000 & Espanha & Pediatras de UTI \\
\hline Albino et al. ${ }^{28}$ & 2002 & Espanha & Medicina de Família \\
\hline Tena et al. ${ }^{29}$ & 2002 & Espanha & Medicina de Família \\
\hline Munoz et al. ${ }^{30}$ & 2003 & Espanha & Medicina de Família \\
\hline Aranda-Beltrán et al. ${ }^{31}$ & 2005 & México & Medicina de Família \\
\hline Beltrán et al. ${ }^{32}$ & 2005 & México & Medicina de Família \\
\hline Palmer-Morales et al. ${ }^{33}$ & 2005 & México & Anestesiologia \\
\hline Beltrán ${ }^{34}$ & 2006 & México & Medicina de Família \\
\hline Tucunduva et al. ${ }^{18}$ & 2006 & Brasil & Oncologia \\
\hline López-León et al. ${ }^{35}$ & 2007 & México & Medicina de Família \\
\hline Barros et al. ${ }^{36}$ & 2008 & Brasil & Plantonistas de UTI \\
\hline Gil-Monte $^{37}$ & 2008 & Argentina & Pediatria \\
\hline Soler et al. ${ }^{38}$ & 2008 & $\begin{array}{c}\text { Bulgária, Croácia, França, Grécia, } \\
\text { Hungria, Itália, Malta, Polônia, } \\
\text { Espanha, Suécia, Turquia e Inglaterra }\end{array}$ & Medicina de Família \\
\hline Grau et al. ${ }^{39}$ & 2009 & $\begin{array}{c}\text { Argentina, México, Equador, Peru, } \\
\text { Colômbia, Uruguai, Guatemala, } \\
\text { Espanha, El Salvador }\end{array}$ & $\begin{array}{l}\text { Clínica geral, Pediatria, Medicina } \\
\text { Interna, Ginecologia, Cirurgia, } \\
\text { Cardiologia, Psiquiatria, Medicina } \\
\text { de Família, Gastroenterologia, } \\
\text { Emergências, Ortopedia, } \\
\text { Anestesiologia, Oftalmologia, } \\
\text { Dermatologia, Infectologia, } \\
\text { Neurologia, Nefrologia. }\end{array}$ \\
\hline Pereda-Torales et al..$^{40}$ & 2009 & México & Medicina de Família \\
\hline Aguilera et al. ${ }^{41}$ & 2010 & México & Medicina de Família \\
\hline Houkes et al. ${ }^{42}$ & 2011 & Holanda & Clínica Geral \\
\hline Roth et al. ${ }^{43}$ & 2011 & $\begin{array}{c}\text { EUA, Canadá, Austrália, Bélgica, } \\
\text { Dinamarca, Finlândia, Islândia, Israel, } \\
\text { Holanda, Noruega, Suécia, Nova } \\
\text { Zelândia e Reino Unido }\end{array}$ & Oncologia Pediátrica \\
\hline Serralheiro et al. ${ }^{44}$ & 2011 & Brasil & Anestesiologia \\
\hline Barbosa et al. ${ }^{45}$ & 2012 & Brasil & Plantonistas de UTI \\
\hline Cubillo et al. ${ }^{46}$ & 2012 & Espanha & Medicina de Família \\
\hline Teixeira et. al. ${ }^{9}$ & 2013 & Portugal & Médicos de UTI \\
\hline Sánchez-Cruz et al. ${ }^{47}$ & 2013 & México & Medicina de Família \\
\hline Galván et al. ${ }^{48}$ & 2014 & Argentina & Pediatria de UTI \\
\hline Garcia et al. ${ }^{49}$ & 2014 & Brasil & Pediatria de UTI e Pediatria Geral \\
\hline Shanafelt et al. ${ }^{50}$ & 2014 & EUA & Oncologia \\
\hline Zanatta et al. ${ }^{51}$ & 2015 & Brasil & Onco-hematologia Pediátrica \\
\hline
\end{tabular}


Ainda não há um consenso na literatura acerca da interpretação do MBI para caracterizar a SB. Há autores $^{52,53}$ que definem a presença da doença pelo critério dos três itens em nível grave, enquanto outros ${ }^{54}$ acreditam que uma dimensão em nível grave, independente de qual seja, é suficiente para diagnosticá-la. Neste estudo, foi considerado a presença dos três itens em nível severo para o diagnóstico da doença, pois, de acordo com a literatura, é necessário que as três dimensões sejam afetadas para conferir maior fidedignidade ao real diagnóstico dessa doença ${ }^{6}$. O resultado da SB nas diferentes especialidades médicas está exposto na Tabela 2. O total de médicos que responderam ao questionário MBI dos artigos selecionados, independente da especialidade, foi de 14.163.

Foi calculada a média ponderada da frequência da SB, levando em consideração a percentagem da doença e o tamanho da amostra de cada pesquisa, e agrupadas nas especialidades correspondentes (Figura 3).

Tabela 2 Resultado da Síndrome de Burnout (SB) nas diferentes especialidades médicas, relacionado com a quantidade de participantes, país do estudo e ano de publicação.

\begin{tabular}{|c|c|c|c|c|}
\hline País & Ano & Especialidades & $N^{o}$ participantes & $S B$ \\
\hline Brasil & 2011 & Anestesiologia & 59 & $3,4 \%$ \\
\hline México & 2005 & Anestesiologia & 89 & $6,9 \%$ \\
\hline Holanda & 2011 & Clínica Geral & $\begin{array}{l}2002=349 \\
2004=261 \\
2006=243\end{array}$ & $\begin{array}{l}2002=20 \% \\
2004=9 \% \\
2006=13 \%\end{array}$ \\
\hline $\begin{array}{l}\text { Argentina; México; Equador; } \\
\text { Peru; Colômbia; Uruguai; } \\
\text { Guatemala; Espanha; } \\
\text { El Salvador; Outros países. }\end{array}$ & 2009 & $\begin{array}{c}\text { Clínica Geral }(20,3 \%) ; \\
\text { Pediatria }(17,1 \%) ; \\
\text { Medicina Interna }(14,7 \%) ; \\
\text { Ginecologia }(6,8 \%) ; \\
\text { Cirurgia }(6,4 \%) ; \\
\text { Cardiologia }(4,9 \%) ; \\
\text { Psiquiatria }(3,5 \%) ; \\
\text { Medicina de família }(2,6 \%) ; \\
\text { Gastroenterologia }(2,3 \%) ; \\
\text { Emergências }(2,2 \%) ; \\
\text { Ortopedia }(2 \%) ; \\
\text { Anestesiologia }(1,8 \%) ; \\
\text { Oftalmologia }(1,6 \%) ; \\
\text { Dermatologia }(1,5 \%) ; \\
\text { Infectologia }(1,5 \%) ; \\
\text { Neurologia }(1,2 \%) ; \\
\text { Nefrologia }(1,2 \%) ; \\
\text { Outras }(8,4 \%)\end{array}$ & 9849 & $\begin{array}{c}\text { Clínica Geral = 11,5\%; } \\
\text { Pediatria = 11,7\%; } \\
\text { Medicina Interna = 15,5\%; } \\
\text { Ginecologia = 10,9\%; } \\
\text { Cirurgia = 12\%; } \\
\text { Cardiologia } 12,6 \% ; \\
\text { Psiquiatria = 7,5\%; } \\
\text { Medicina de Família = 12\%; } \\
\text { Gastroenterologia }=12,3 \% ; \\
\text { Emergências = } 17 \% ; \\
\text { Ortopedia }=14,5 \% ; \\
\text { Anestesiologia }=5 \% ; \\
\text { Oftalmologia }=10,3 \% ; \\
\text { Dermatologia }=5,3 \% ; \\
\text { Infectologia }=14,3 \% ; \\
\text { Neurologia }=8,9 \% ; \\
\text { Nefrologia }=12,8 \% .\end{array}$ \\
\hline $\begin{array}{l}\text { Bulgária, Croácia, França, } \\
\text { Grécia, Hungria, Itália, Malta, } \\
\text { Polônia, Espanha, Suécia, } \\
\text { Turquia e Inglaterra. }\end{array}$ & 2008 & Medicina de Família & 1390 & $12 \%$ \\
\hline Espanha & 2002 & Medicina de Família & 586 & $42,5 \%$ \\
\hline México & 2005 & Medicina de Família & 197 & $2 \%$ \\
\hline México & 2007 & Medicina de Família & 131 & $1,5 \%$ \\
\hline México & 2013 & Medicina de Família & 130 & $6 \%$ \\
\hline Portugal & 2013 & Médicos de UTI & 82 & $22 \%$ \\
\hline Brasil & 2015 & Onco-hematologia Pediátrica & 36 & $5,6 \%$ \\
\hline Brasil & 2006 & Oncologia & 136 & $3 \%$ \\
\hline Argentina & 2008 & Pediatria & 123 & $10,6 \%$ \\
\hline Espanha & 2000 & Pediatria de UTI & 68 & $10 \%$ \\
\hline Brasil & 2014 & $\begin{array}{l}\text { Pediatria de UTI } \\
\text { e Pediatria Geral }\end{array}$ & $\begin{array}{c}35 \text { UTI; } \\
35 \text { Gerais }\end{array}$ & $\begin{array}{l}\mathrm{UTI}=17 \% \\
\text { Gerais }=0 \%\end{array}$ \\
\hline Brasil & 2008 & Plantonistas de UTI & 297 & $7,4 \%$ \\
\hline Brasil & 2012 & Plantonistas de UTI & 67 & $17,91 \%$ \\
\hline
\end{tabular}




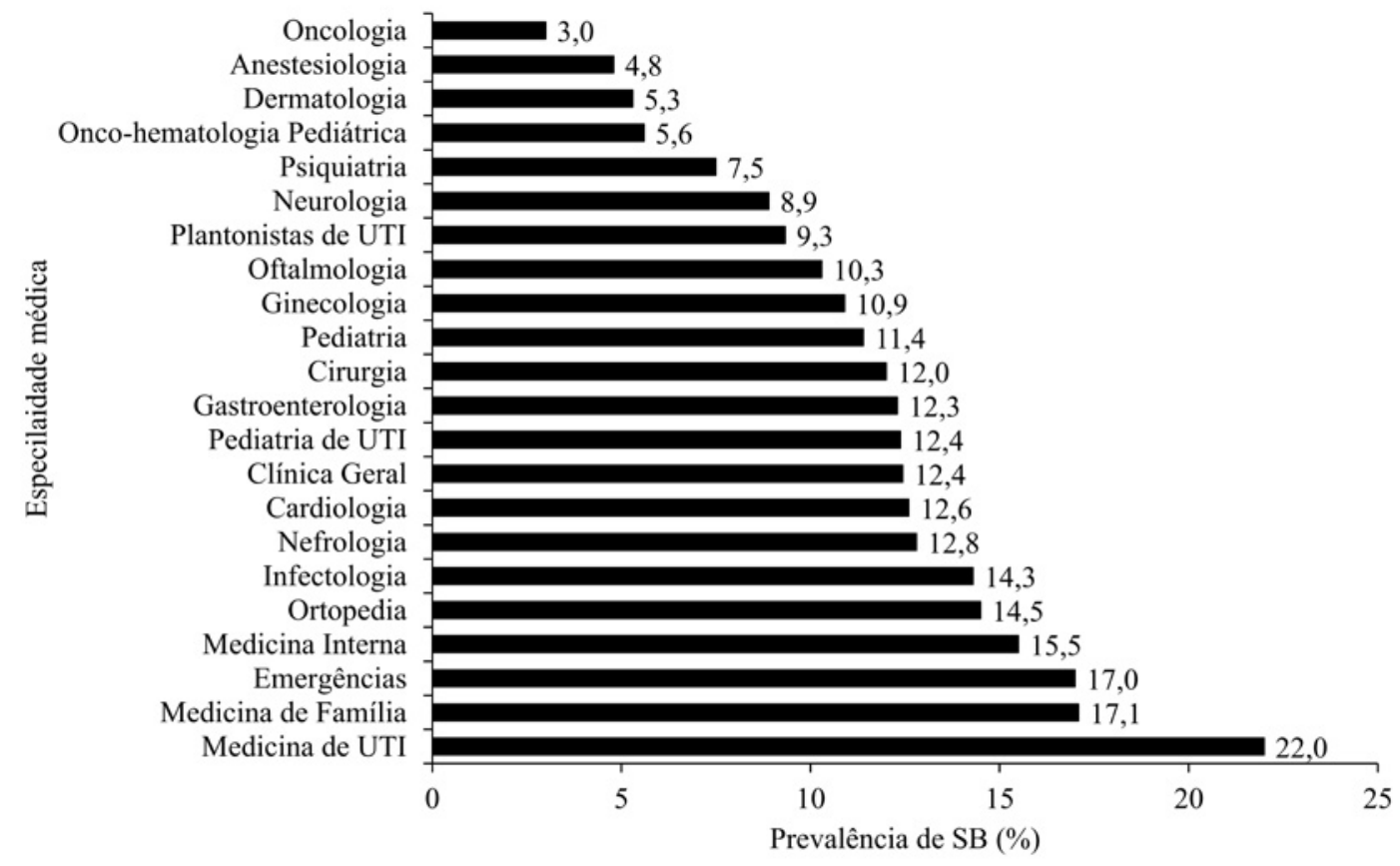

Figura 3 Prevalência da Síndrome de Burnout (SB) nas especialidades médicas

\section{Discussão}

Segundo a literatura ${ }^{3,55,56}$, a etiologia do desenvolvimento da SB é multifatorial. Neste contexto, Gil-Monte e Peiró ${ }^{55}$, ao discutirem sobre a etiologia da síndrome, classificaram os diversos fatores em facilitadores e desencadeadores. Foi observado, por meio dos estudos, que cada especialidade médica está exposta a diferentes fatores desencadeadores e facilitadores que influenciam na instalação da SB. Os desencadeadores são fatores extenuantes contínuos no ambiente de trabalho, e os facilitadores são situações relativas à pessoa que podem agir como preditores ou inibidores da atividade do stress sobre ela ${ }^{3,55,56}$.

Sendo assim, a discussão discorrerá sobre as cinco maiores e as cinco menores frequências da SB deste trabalho, em que são expostos os fatores presentes em cada especialidade.

Os médicos de Unidade de Terapia Intensiva (UTI) apresentaram a maior frequência da síndrome. A idade avançada e muitos anos de experiência profissional na UTI atuam como fatores desencadeadores da doença. Um item do MBI que apresentou significância estatística foi a exaustão emocional, relacionada aos conflitos éticos e profissionais que acarretam stress. Por exemplo: não ter um contrato fixo de trabalho, assistir doentes graves, tomar a decisão de suspender o tratamento e proceder à sedação terminal. Também foram relacionados os conflitos pessoais com os colegas ou a família do enfermo. Dessa maneira, é necessária uma preparação psicológica específica no decurso da especialização para possibilitar melhor gerenciamento do stress diário destes profissionais. Entretanto, trabalhar em outras áreas do hospital é um fator protetor para a SB. Além disso, a divisão multidisciplinar adequada das atividades na UTI propicia a distribuição das responsabilidades e atenua a tensão profissional no médico $^{9,36,45}$. Os médicos de família (MF) e os médicos de emergência (ME) apresentaram a segunda e a terceira maior frequência, respectivamente, da SB. Observamos que para ambos foi encontrada relação entre o burnout de alto grau e elevado uso de tabaco, álcool ou medicação psicotrópica, entendidos como manifestações de baixa autoestima ${ }^{36,56}$. Os fatores identificados como desencadeadores de stress contínuo foram a inadequada comunicação institucional e a burocrática organização multidisciplinar do trabalho, a falta de apoio dos cargos superiores, a insatisfação com a carreira e o salário, sendo este associado à alta despersonalização $26,28,30,34,35,38,46$.

Há aspectos que são peculiares aos MF e aos ME. Foi notado que o pico da prevalência da SB ocorre nos ME que trabalham de 13 a 15 turnos/mês, e naqueles com mais de 16 turnos/mês houve queda significativa da frequência da doença, explicada pelo processo dinâmico do desenvolvimento da SB em diferentes indivíduos, em que há profissionais que adquirem habilidades de enfrentamento bem-sucedido com o passar do tempo. Foi constatada a associação entre alta taxa da SB e baixo grau de 
envolvimento com o trabalho. Não foram encontradas relações com idade, gênero, estado civil e nível de qualificação profissional ${ }^{26}$.

Com relação aos MF, foram observados como fatores desencadeadores da SB: a alta carga de trabalho ou turnos extras ${ }^{40,46,47}$, expectativa não realista do trabalho e falta de recursos, como a escassez de instrumentos na atenção primária ${ }^{29,35,40}$. Não há consenso sobre o papel da idade avançada nos MF, pois foram constatados trabalhos que a atribuem como fator protetor $^{29,38,47}$, outros que a analisam como fator desencadeador $^{28,40,41}$ e os que não encontraram nenhuma relação ${ }^{34,35}$. A associação da doença com o gênero e com o estado civil também é confusa, fato que impede conclusões a respeito. Contudo, o nível de qualificação profissional foi um desencadeador determinante, em que os MF com níveis de estudo discrepante ao posto que ocupam foram relacionados com alta exaustão emocional ${ }^{31,36}$. No que diz respeito à medicina interna, especialidade analisada com o quarto maior número de casos, foram constatados como fatores desencadeadores o contato contínuo do médico com o sofrimento e a morte, o impacto negativo do stress ocupacional sobre a vida familiar e a falta de estímulo intelectual do trabalho. Também foi observado que a insatisfação nas relações mantidas com os pacientes e suas famílias, devido à sobrecarga de trabalho, tem impacto negativo nas três dimensões da $\mathrm{SB}^{31}$. Além disso, houve correspondência estatisticamente significativa entre a presença da síndrome em alto nível e os portadores de doenças crônicas e afastados do serviço por motivos de saúde. A idade, a satisfação no trabalho, o perfil otimista e a percepção de ser valorizado foram fatores protetores ${ }^{39}$.

A quinta maior prevalência ocorreu entre os ortopedistas. Foram observados os seguintes fatores desencadeadores: pouco tempo para recreação depois de dias de alta demanda de trabalho, associado à fadiga; exigências laborais físicas e psicológicas, muitas vezes com privação do sono, repercutindo na necessidade/dependência de medicamentos psicotrópicos. O salário percebido como baixo pode ser o estímulo a trabalhos em turnos extras, aumentando o stress, consequentemente, causando desgaste emocional. Não obstante, a motivação profissional agiu como fator protetor, em que foram relacionados fatores como a necessidade de contato com pacientes, o interesse em ajudar o próximo e o aumento salarial condizente com o trabalho excessivo ${ }^{57}$.

Foi analisado o grupo das cinco especialidades com menores frequências da SB. Dentre estes, foi constatado a maior prevalência em psiquiatras. Os fatores protetores foram relacionados às recompensas referentes ao trabalho, em que há o reconhecimento profissional pelos pacientes assistidos, é correspondida a expectativa profissional dos médicos, há satisfação na escolha da psiquiatria, é agradável a estrutura física e é possível haver formação contínua. Também foi notado que o horário estável de trabalho possibilita momentos de descanso. Contudo, a alta demanda de pacientes, a saída do local de trabalho para atender a chamadas, os prazos curtos para realizar muitas tarefas e a alta burocracia do sistema foram fatores desencadeadores de stress ${ }^{58}$.

Foi obtido o quarto menor número de casos da SB em médicos que prestam cuidados aos pacientes onco-hematológicos infantis. Foram examinados os fatores protetores, que são as recompensas psicológicas fortemente ligadas à profissão médica, como aliviar a dor, ter competência para diagnosticar corretamente, salvar vidas, prevenir doenças e o reconhecimento dos assistidos. Além disso, no hospital da pesquisa, o índice de cura do câncer e doenças hematológicas, de crianças e adolescentes, foi de aproximadamente $80 \%$. Isso faz com que os oncologistas sintam-se orgulhosos e tenham uma alta realização profissional. Entretanto, houve associação significativa entre a alta exaustão emocional e problemas de saúde relacionados ao trabalho, em que os mais frequentes são lombalgias, stress e depressão. Ainda assim, foram analisados na amostra alguns profissionais cujo trabalho é terceirizado, fato que produz o sentimento de desvalorização pelo vínculo precário com a instituição, o que reflete em baixa realização profissional ${ }^{51}$.

Em relação aos anestesistas e dermatologistas, segunda e terceira especialidades, respectivamente, com menor frequência da doença, foi constatado que há influência dos países onde residem e a SB, sendo que foi observado menor prevalência em nações economicamente menos desenvolvidas. Isso ocorre porque o contexto laboral nessas localidades faz com que haja menor stress nos profissionais, pois os doentes tendem a ser menos esperançosos e menos exigentes. Além disso, foi analisado que o paradigma socioeconômico local propicia o desenvolvimento de outros transtornos mentais e físicos em detrimento da $\mathrm{SB}^{39}$. Entretanto, a escassez de estudos em anestesistas é um fator a ser considerado nos resultados obtidos $^{33,44}$.

Já em oncologistas, foi encontrada a menor frequência da SB, em que a prática de exercícios físicos, de atividades recreativas ou trabalhar exclusivamente em instituições públicas foram associados a um menor nível de exaustão emocional ${ }^{18}$. Além disso, foi observado ${ }^{59}$ relação entre mulheres e maior taxa da SB, e na amostra deste trabalho há $82,8 \%$ oncologistas do sexo masculino, dado que pode influenciar na prevalência ${ }^{50}$. Nos oncologistas da amostra, a média de idade foi de 53,6 anos, resultando em um fator de proteção ao surgimento da SB. Segundo a opinião dos próprios oncologistas, 
as condições mais importantes que atuam para evitar a instalação da síndrome são: reduzir a burocracia, limitar a quantidade de doentes atendidos diariamente $^{50}$ e incrementar a educação continuada, tanto em quantidade quanto em qualidade ${ }^{18}$.

Logo, há fatores relacionados à SB comuns às especialidades, sendo analisados os que agem como desencadeadores. A falta de contrato fixo de trabalho é uma condição que acarreta incerteza sobre o futuro do profissional na instituição. Por isso, é necessário que haja o vínculo contratual para aumentar sua valorização e reduzir o número de conflitos, a permitir que os médicos foquem suas atenções na qualidade do atendimento e aumentem o grau de envolvimento com o trabalho. A comunicação precária da instituição também é um fator de stress contínuo, que dificulta, por exemplo, sugestões de melhoria da burocracia organizacional por meio dos profissionais que estão no dia a dia e conhecem a realidade. Também há relação entre o burnout de alto grau e alto consumo de tabaco, álcool ou medicação psicotrópica. Ainda assim, o alto número de pacientes a ser atendidos gera uma sobrecarga que promove exaustão emocional e pode ser a causa de doenças sistêmicas crônicas. Logo, é necessário distribuir os atendimentos de modo multidisciplinar, de acordo com o nível de complexidade de cada caso. A medicina preventiva é a solução mais plausível, mas o investimento na atenção primária demonstra ainda ser pouco valorizada. Aumentar a quantidade de médicos no serviço também é uma solução, mas deve-se considerar que pode não haver profissionais suficientes no mercado de trabalho, assim como analisar se os gastos com novos contratos são mais eficientes em relação aos gastos com o afastamento do trabalho em decorrência da $\mathrm{SB}^{3,5,9,20,21,26,28-31,34-36,38,40,46,47,54,56,57}$.

Foram averiguados os fatores protetores que, de maneira geral, estão relacionados à personalidade de cada indivíduo e às recompensas profissionais. Os médicos com perfil otimista sentem-se mais valorizados e satisfeitos com a profissão. A motivação também é importante, que pode ser promovida pelo aumento salarial, condizente com a carga extra de trabalho, o reconhecimento e estímulo da instituição por meio da educação continuada, a garantir maior segurança na atuação e aumentar a realização profissional. Dessa maneira, o processo de instalação da SB é dinâmico, em que o indivíduo passa por diferentes estágios até que a doença realmente se instale, sendo possível preveni-la antes que os profissionais desenvolvam o nível mais grave e, por vezes, irremediável. A psicoterapia em grupo é um método terapêutico que auxilia no enfrentamento do stress, sendo um evento constante no transcurso de toda a vida do médico. O exercício físico é outro meio efetivo de redução do efeito do stress sobre o indivíduo, assim como a prática de atividades recreativas que tragam prazer $18,39,51,57,58$.

Porém, a relação entre algumas variáveis e a SB não foi bem esclarecida, como a idade, o gênero, o estado civil, a carga horária e aexperiência laboral. Também houve disparidade em relação ao número de estudos feitos em diferentes especialidades médicas e falta de padronização ao definir a SB devido a diferentes critérios de interpretação dos itens do MBI, o que interferiu na análise final.

\section{Conclusão}

As especialidades analisadas com as maiores frequências da SB foram respectivamente: Medicina de UTI, Medicina de Família, Medicina de Emergências, Medicina Interna e Ortopedia. Os fatores associados em destaque foram os relacionados à organização e estrutura do trabalho e a maneira de enfrentamento ao stress. As áreas menos afetadas, em ordem decrescente, foram: Psiquiatria, Onco-hematologia Pediátrica, Dermatologia, Anestesiologia e Oncologia, em que se ressaltam os fatores organizacionais, o contexto regional de trabalho e as recompensas emocionais.

\section{Contribuição dos autores}

Todos os autores contribuíram substancialmente no delineamento, no levantamento de dados e na sua análise e interpretação, e na elaboração ou aprovação da versão final do manuscrito.

\section{Referências}

1. Freudenberger HJ. Staff burn-out. J Soc Issues. 1974;30(1):159-65.

2. Maslach C, Jackson SE. The measurement of experienced burnout. J Occup Behav. 1981;2:99-113.
3. Maslach C, Leiter MP. [tradução Martins MS]. Trabalho: fonte de prazer ou desgaste? Guia para vencer o estresse na empresa. Campinas: Papirus; 1999. 
4. Silva DC, Loureiro MF, Peres RS. Burnout em profissionais de enfermagem no contexto hospitalar. Psicol Hosp. 2008;6(1):39-51.

5. Dias S, Queirós C, Carlotto MS. Síndrome de burnout e fatores associados em profissionais da área da saúde: um estudo comparativo entre Brasil e Portugal. Aletheia. 2010;32:4-21.

6. Maslach C, Jackson SE, Leiter MP. The Maslach Burnout Inventory manual. 3rd ed. Palo Alto, CA: Consulting Psychologists Press; 1996.

7. Maslach C, Schaufeli WB, Leiter MP. Job burnout. Annu Rev Clin Psychol. 2001;52:397-422.

8. Carlotto MS, Camara SG. Propriedades psicométricas do Maslach Burnout Inventory em uma amostra multifuncional. Estud Psicol (Campinas). 2007;24(3):325-32.

9. Teixeira C, Ribeiro O, Fonseca AM, Carvalho AS. Burnout in intensive care units - a consideration of the possible prevalence and frequency of new risk factors: a descriptive correlational multicenter study. BMC Anesthesiol. 2013;13(1):38.

10. Organização Mundial da Saúde. CID-10 Classificação Estatística Internacional de Doenças e Problemas Relacionados à Saúde [Internet]. 2008 [acesso em 2017 fev 14]. Disponível em: http:// www.datasus.gov.br/cid10/V2008/v2008.htm.

11. Carlotto MS, Câmara SG. Análise da produção científica sobre a Síndrome de Burnout no Brasil. PSICO. 2008;39(2):152-8.

12. Benevides-Pereira AMT. O estado da arte do Burnout no Brasil. Rev Eletrônica InterAção Psy [online]. 2003;1(1):4-11 [acesso em $2017 \mathrm{fev} 14$ ]. Disponível em: http:/www.saudeetrabalho.com.br/ download_2/burnout-benevides.pdf.

13. Trigo TR, Teng CT, Hallak JE. Síndrome de burnout ou estafa profissional e os transtornos psiquiátricos. Rev Psiquiatr Clín. 2007;34(5):223-33.

14. Creagan ET. Stress among medical oncologists: the phenomenon of burnout and a call to action. Mayo Clin Proc. 1993;68(6):614-5.

15. Carlotto MS, Gobbi MD. Síndrome de burnout: um problema do indivíduo ou do seu contexto de trabalho? Aletheia. 1999;10:103-14.

16. Gil-Monte PR. Validez factorial de la adaptación al español del Maslach Burnout Inventory: general survey. Salud Pública Méx. 2002;44(1):33-40.

17. González RP, González JF. Prevalencia del síndrome de burnout o desgaste profesional en los médicos de atención primaria. Aten Primaria. 1998;22(9):580-4.

18. Tucunduva LT, Garcia AP, Prudente FV, Centofanti G, Souza CM, Monteiro TA, et al. A síndrome da estafa profissional em médicos cancerologistas brasileiros. Rev Assoc Med Bras. 2006;52(2):108-12.

19. Barbosa GA. A saúde dos médicos no Brasil. Brasília: Conselho Federal de Medicina; 2007.

20. Lima RA, Souza AI, Galindo RH, Feliciano KV. Vulnerabilidade ao burnout entre médicos de hospital público de Recife. Cien Saude Colet. 2013;18(4):1051-8.

21. Rosa C, Carlotto MS. Síndrome de Burnout e satisfação no trabalho em profissionais de uma instituição hospitalar. Rev SBPH (online). 2005;8(2):1-15.

22. SRCOM/Projeto de prevenção da Síndrome de 'Burnout' [Internet]. Rev SRCOM. 2015;(2):27-32 [acesso em 2015 nov 11]. Disponível em: http:// omcentro.com/ficheiros/docs/versaoweb_srcom_ revista.pdf.

23. Gonçalves TB, Leitão AK, Botelho BS, Marques RA, Hosoume VS, Neder PR. Prevalência de síndrome de burnout em professores médicos de uma universidade pública em Belém do Pará [Internet]. Rev Para Med. 2012;26(4) [acesso em 2015 nov 1]. Disponível em: http://files.bvs.br/ upload/S/0101-5907/2012/v26n4/a3483.pdf.

24. Liberati A, Altman DG, Tetzlaff J, Mulrow C, Gøtzsche PC, Ioannidis JPA, et al. The PRISMA statement for reporting systematic reviews and meta-analyses of studies that evaluate healthcare interventions: explanation and elaboration. BMJ. 2009;339:b2700. PubMed PMID: 19622552.

25. Moher D, Liberati A, Tetzlaff J, Altman DG, PRISMA Group. Preferred reporting items for systematic reviews and meta-analyses: the PRISMA statement. Ann Intern Med. 2009;151(4):264-9. PubMed PMID: 19622511.

26. Goldberg R, Boss RW, Chan L, Goldberg J, Mallon WK, Moradzadeh D, et al. Burnout and its correlates in emergency physicians: four years' experience with a Wellness Booth. Acad Emerg Med. 1996;3(12):1156-64.

27. Arriortua AB, López-Herce Cid J, Carrillo Álvarez A, Vigil Escribano MD, Lucas García N, Panadero Carlavilla E. Situación de burnout de los pediatras intensivistas españoles. An Pediatr (Barc). 2000;52(5):418-23.

28. Albino LP, Aguero ER, Martínez LM, Vega ED. Burnout en médicos de atención primaria de la província de Cáceres. Aten Primaria. 2002;29(5):294-302.

29. Tena PS, Soriano JS, Bernal JS, Mulet EM, Muñoz CR, García M, et al. Desgaste profesional en los médicos de atención primaria de Barcelona. MEDIFAM. 2002;12(10):613-19.

30. Martínez de la Casa Muñoz A, Castillo Comas C, Magaña Loarte E, Bru Espino IM, Franco Moreno A, Segura Fragoso A. Estudio sobre la prevalencia del burnout en los médicos del área sanitaria de Talavera de la Reina. Aten Primaria. 2003;32(6):343-8.

31. Aranda-Beltrán C, Pando-Moreno M, Torres-López T, Salazar-Estrada J, Franco-Chávez S. Factores psicosociales y síndrome de burnout en médicos de familia. México. An Fac Med. 2005;66(3):225-231.

32. Aranda-Beltrán C, Pando-Moreno M, SalazarEstrada JG, Torres-López TM, Aldrete Rodríguez MG, Pérez Reyes MB. Síndrome de burnout en 
médicos familiares del Instituto Mexicano del Seguro Social, Guadalajara, México [Internet]. Rev Cub Salud Publica. 2005;31(2) [acesso em 2015 nov 1]. Disponível em: http://www.bvs.sld.cu/ revistas/spu/vol31_2_05/sp05205.htm.

33. Palmer-Morales L, Gómez-Vera A, Cabrera-Pivaral C, Prince-Velez R, Searcy-Bernal R. Prevalencia del síndrome de agotamiento profesional en médicos anestesiólogos de la ciudad de Mexicali. Gac Med Mex. 2005;141(3):181-83.

34. Beltrán CA. Diferencias por sexo, síndrome de burnout y manifestaciones clínicas, en los médicos familiares de dos instituciones de salud, Guadalajara, México. Rev Costarric Salud Pública. 2006;15(29):1-7.

35. López-León E, Rodríguez-Moctezuma J, LópezCarmona J, Peralta-Pedrero M, Munguía-Miranda C. Desgaste profesional en médicos familiares y su asociación con factores sociodemográficos y laborales. Rev Med Inst Mex Seguro Soc. 2007;45(1):13-19.

36. Barros DS, Tironi MO, Nascimento Sobrinho C, Neves FS, Bitencourt AG, Almeida AM, et al. Médicos plantonistas de unidade de terapia intensiva: perfil sócio-demográfico, condições de trabalho e fatores associados à síndrome de burnout. Rev Bras Ter Intensiva. 2008;20(3):235-40.

37. Gil-Monte P, Marucco MA. Prevalencia del "síndrome de quemarse por el trabajo" (burnout) en pediatras de hospitales generales. Rev Saude Publica [online]. 2008;42(3):450-6.

38. Soler JK, Yaman H, Esteva M, Dobbs F, Asenova RS, Katic M, et al. Burnout in European family doctors: the EGPRN study. Fam Pract. 2008;25(4):245-65.

39. Grau A, Flichtentrei D, Suner R, Prats M, Braga F. Influencia de factores personales, profesionales y transnacionales en el síndrome de burnout en personal sanitario hispanoamericano y español. Rev Esp Salud Publica. 2009;83(2):215-30.

40. Pereda-Torales L, Celedonio FG, Vásquez MT, Zamora MI. Síndrome de burnout en médicos y personal paramédico. Salud Ment. 2009;32(5):399-404.

41. Aguilera EC, García JE. Prevalencia del síndrome de agotamiento profesional (burnout) en médicos familiares mexicanos: análisis de factores de riesgo. Rev Colomb Psiquiatr. 2010;39(1):67-84.

42. Houkes I, Winants Y, Twellaar M, Verdonk P. Development of burnout over time and the causal order of the three dimensions of burnout among male and female GPs. A three-wave panel study. BMC Public Health. 2011;11:240.

43. Roth M, Morrone K, Moody K, Kim M, Wang D, Moadel A, et al. Career burnout among pediatric oncologists. Pediatr Blood Cancer. 2011;57(7):1168-73.

44. Serralheiro FC, Braga AL, Garcia ML, Grigio T, Martins LC. Prevalência da síndrome de burnout em anestesiologistas de Instituição de Ensino Superior em Medicina. Arq Bras Ciênc Saúde. 2011;36(3):140-3.
45. Barbosa FT, Leão BA, Tavares GM, Santos JG. Burnout syndrome and weekly workload of on-call physicians: cross-sectional study. Sao Paulo Med J. 2012;130(5):282-8.

46. Cubillo AC, Guevara JC, Bravo JJ, Riguera MJ, Castro ML, Sanz AG. Evolución del burnout y variables asociadas en los médicos de atención primaria. Aten Primaria. 2012;44(9):532-39.

47. Sánchez-Cruz J, Mugártegui-Sánchez S. Síndrome de agotamiento profesional en los médicos familiares. Rev Med Inst Mex Seguro Soc. 2013;51(4):428-31.

48. Galván ME, Vasallo JC, Rodríguez SP, Otero P, Montonati MM, Cardigni G, et al. Síndrome de desgaste profesional (burnout) en médicos de unidades de cuidados intensivos pediátricos en la Argentina. Rev Soc Bol Ped.2014;53(1):29-36.

49. Garcia TT, Garcia PC, Molon ME, Piva JP, Tasker RC, Branco RG, et al. Prevalence of burnout in pediatric intensivists: an observational comparison with general pediatricians. Pediatr Crit Care Med. 2014;15(8):347-53.

50. Shanafelt TD, Gradishar WJ, Kosty M, Satele D, Chew H, Horn L, et al. Burnout and career satisfaction among US oncologists. J Clin Oncol. 2014;32(7):678-86.

51. Zanatta AB, Lucca SR. Prevalência da síndrome de burnout em profissionais da saúde de um hospital oncohematológico infantil. Rev Esc Enferm USP. 2015;49(2):253-60.

52. Ramirez AJ, Graham J, Richards MA, Cuff A, Gregory WM, Leaning MS, et al. Burnout and psychiatric disorder among cancer clinicians. Br J Cancer. 1995;71(6):1263-69.

53. Ramalho MAN, Nogueira-Martins MCF. Vivências de profissionais de saúde da área de oncologia pediátrica. Psicol Estud [online]. 2007;12(1):123-32.

54. Grunfeld E, Whelan TJ, Zitzelsberger L, Willan AR, Montesanto B, Evans WK. Cancer care workers in Ontario: prevalence of burnout, job stress and job satisfaction. CMAJ. 2000;163(2):166-9.

55. Gil-Monte P, Peiró JM. Desgaste psíquico en el trabajo: el síndrome de quemarse. Madrid: Sintesis; 1997.

56. Borges LO, Argolo JC, Pereira AL, Machado EA, Silva WS. A síndrome de burnout e os valores organizacionais: um estudo comparativo em hospitais universitários. Psicol Refl Crít. 2002;15(1):189-200.

57. Mello MH, Souza JC. Quality of life of orthopedists in Mato Grosso do Sul. Rev Bras Ortop. 2013;48(1):92-9.

58. Tejada MP, Gomez OV. Factores psicosociales y laborales asociados al burnout de psiquiatras en Colombia. Rev Colomb Psiquiatr. 2009;38(3):488-512.

59. McMurray JE, Linzer M, Konrad TR, Douglas J, Shugerman R, Nelson K. The work lives of women physicians results from the physician work life study. J Gen Intern Med 2000;15(6):372-80. 